\title{
Measurements of short-lived fission product yields using monoenergetic neutron and photon beams
}

\author{
Sean Finch ${ }^{1,2,3, *}$, Matthew Gooden ${ }^{4}$, Chris Hagmann ${ }^{3}$, Calvin Howell ${ }^{1,2}$, Vanessa Linero ${ }^{4,5}$, \\ Jack Silano $^{3}$, Mark Stoyer ${ }^{3}$, Anton Tonchev ${ }^{1,3}$, Werner Tornow ${ }^{1,2}$, Innocent Tsorxe ${ }^{2,6}$, and \\ Jerry Wilhelmy ${ }^{4}$ \\ ${ }^{1}$ Duke University, Durham, NC 27708 \\ ${ }^{2}$ Triangle Universities Nuclear Laboratory, Durham, NC 27708 \\ ${ }^{3}$ Lawrence Livermore National Laboratory, Livermore, CA 94550 \\ ${ }^{4}$ Los Alamos National Laboratory, Los Alamos, NM 87544 \\ ${ }^{5}$ Colorado School of Mines, Golden, CO 80401 \\ ${ }^{6}$ North Carolina State University, Raleigh, NC 27695
}

\begin{abstract}
A joint TUNL-LLNL-LANL collaboration was formed to measure the absolute fission product yields from the ${ }^{235} \mathrm{U},{ }^{238} \mathrm{U}$, and ${ }^{239} \mathrm{Pu}$ isotopes. Our goal is to study the energy evolution of fission products using monoenergetic beams. In order to extend our successful fission product-yield studies to include products with shorter half-lives, a RApid Belt-driven Irradiated Target Transfer System, named RABITTS, was constructed. This system allows us to perform cyclic activation and quantify fission products with $\gamma$-ray spectroscopy using HPGe detectors. Both a 1 meter and 10 meter transfer system have been developed, with transit times of 0.4 and 1.1 seconds, respectively. Using these systems, we have measured sub-second half-lives. Our goal is to measure fission product yields from neutron-induced fission with $E_{n}=0.5-14.8 \mathrm{MeV}$ and photofission with $E_{\gamma}=8-15 \mathrm{MeV}$. A detailed characterization of the system's performance is presented, including preliminary fission product measurements, and the expected sensitivity.
\end{abstract}

\section{Introduction}

Fission product yields (FPYs) are a principal observable of the fission process. The dominant nuclear fission process produces two fission fragments, which promptly evaporate neutrons and result in two fission products. These fission products are created far from nuclear stability, and usually undergo multiple decays before reaching stability. The FPY is the percentage production of a given fission product. Independent FPYs are the initial product yields following post scission neutron emission. The cumulative FPY of a particular isotope is the sum of its independent yield plus the independent yields of all unstable precursor isotopes that $\beta$ decay into that isotope, as shown in Fig. 1.

An experimental advantage of FPYs is that they provide fundamental fission information that can be obtained using radiochemical or counting techniques at a time that is delayed relative to the prompt fission event. A joint TUNL-LLNL-LANL collaboration has produced

*e-mail: sfinch@tunl.duke.edu 
such measurements of absolute FPYs [1,2], primarily focusing on cumulative yields. The experimental goal of this program is to measure the energy dependence of FPYs as a function of incident neutron energies from $E_{n}=0.5$ to $14.8 \mathrm{MeV}$ using monoenergetic beams [2]. Most FPY evaluations only consider three incident neutron energies: thermal, fission spectrum $(\approx 1.5 \mathrm{MeV})$, and $14.8 \mathrm{MeV}[3]$. We are also pursuing additional measurements to include photofission product yields at $E_{\gamma}=8-15 \mathrm{MeV}$ [4]. The neutron induced fission measurements are performed on the main three actinides: ${ }^{235} \mathrm{U},{ }^{238} \mathrm{U}$, and ${ }^{239} \mathrm{Pu}$, using the same facilities and techniques. Great detail is taken to perform these experiments in such a way as to minimize systematic uncertainties between the different measurements. These results are an extensive self-consistent nuclear data set of FPYs.

The goal of the present work is to extend these cumulative FPY measurements towards independent FPY measurements. This involves measuring fission products with progressively shorter half-lives. The technique to be used for these measurements is detailed in Sec. 1.1. and preliminary fission spectra are presented in Sec. 2.1.

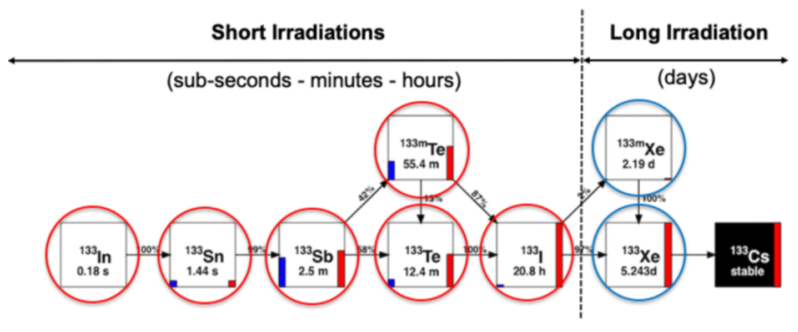

Figure 1. The $\mathrm{A}=133$ isobar, illustrating the possible fission products. The cumulative FPY of the entire $A=133$ isobaric chain is measured using the yield of ${ }^{133} \mathrm{Xe}$. Additional measurements of the shorter-lived fission products will be able to provide information on the independent yields.

\subsection{Experimental technique}

Quasi-monoenergetic neutron beams were produced at Triangle Universities Nuclear Laboratory's (TUNL) $10 \mathrm{MV}$ tandem accelerator using the ${ }^{7} \mathrm{Li}(p, n){ }^{7} \mathrm{Be},{ }^{3} \mathrm{H}(p, n){ }^{3} \mathrm{He},{ }^{2} \mathrm{H}(d, n){ }^{3} \mathrm{He}$, and ${ }^{3} \mathrm{H}(d, n)^{4} \mathrm{He}$ reactions. For the photofission studies, the High Intensity $\gamma$-ray Source $(\mathrm{HI} \gamma \mathrm{S})[5]$ was used. $\mathrm{HI} \gamma \mathrm{S}$ produces monoenergetic $\gamma$ rays in the energy range from 2 to $100 \mathrm{MeV}$ from the Compton back-scattering of free electron laser light in an electron storage ring. The typical $\gamma$-ray flux delivered on targets for this work was $4 \times 10^{8} \gamma /\left(\mathrm{cm}^{2} \mathrm{~s}\right)$.

FPYs are determined via the activation technique: following irradiations, the targets are $\gamma$ ray counted using high purity germanium (HPGe) detectors. The fission products are tracked over multiple half-lives. This allows for precise measurement of the fission product's characteristic $\gamma$-ray energy spectra and half-life, which helps to unambiguously identify the exact fission product. The total number of fissions in the activation target is determined using a dual fission chamber, and mass scaling the thin fission chamber foils to the thick activation target. The power of this technique is that it does not rely on measurement of the exact beam flux or any reference cross-section data. The technique is characterized in Ref. [6], and updated Monte-Carlo simulations of the target geometry have been developed for the present work.

The original measurements focused primarily on cumulative FPYs [1, 2, 4]. These measurements, as shown in the right side of Fig. 1, typically used 3-5 day long irradiations, followed by 1-2 month counting cycles, and resulted in measurements of 16 FPYs with 
$t_{1 / 2}>0.5 \mathrm{~h}$. Shorter $1 \mathrm{~h}$ long irradiations were then performed to measure yields of an additional 45 fission products with $t_{1 / 2}>3 \mathrm{~min}$. The minimum observable $t_{1 / 2}$ is limited by the transit time from the activation source to the low background counting stations located in an adjacent building. In order to reduce the delay time between the end of target irradiation and the beginning of counting to the shortest possible value, a new experimental capability has been added to TUNL: a RApid Belt-driven Irradiated Target Transfer System (RABITTS).

\subsection{RABITTS}

RABITTS is a fully automatic system that moves a target between irradiation and counting positions in order to perform cyclic activation $[7,8]$. The user may program irradiation times, counting times, and transit speeds. FPYs have previously been measured using cyclic activation and a fast transit system at $E_{n}=14.8 \mathrm{MeV}$ [9]. A total of three RABITTS were constructed: a $1 \mathrm{~m}$ and a $10 \mathrm{~m}$ track for neutron-induced fission, and a $1 \mathrm{~m}$ track for photofission studies. These three systems are identical except for track length and installation location. RABITTS has a maximum speed of $10 \mathrm{~m} / \mathrm{s}$, which results in a $0.4 \mathrm{~s}$ transit time for the 1 meter systems, and a $1.1 \mathrm{~s}$ transit time for the $10 \mathrm{~m}$ system.

The RABITTS is powered by a servo motor attached to a belt-driven linear actuator track. The servo motor and linear actuator has a position repeatability of $34 \mu \mathrm{m}$ and $25 \mu \mathrm{m}$, respectively. This combined system results in a $42 \mu \mathrm{m}$ repeatability for our target sample. Cyclic activation has traditionally been performed using pneumatic systems. The present approach, using a servo motor, track, and belt, was chosen for its high repeatability, controlled soft acceleration and deceleration. Furthermore, there are no restrictions on the target mass, size, or shape, and the target remains in a consistent orientation throughout the cycle. For the present experiments, thin targets with a total mass of 400-500 $\mathrm{mg}$ have been utilized. The use of these relatively thin targets minimizes corrections such as multiple scatter effects and self $\gamma$-ray attenuation.

A schematic of the $10 \mathrm{~m}$ RABITTS is shown in Fig. 2. The actinide target is irradiated in the center of a large $10 \mathrm{~m} \times 10 \mathrm{~m}$ room, then transported through a thick concrete shielding wall to a low background counting area. In the present work, two HPGe detectors are used, with the capability to include two additional HPGe detectors during future experiments.

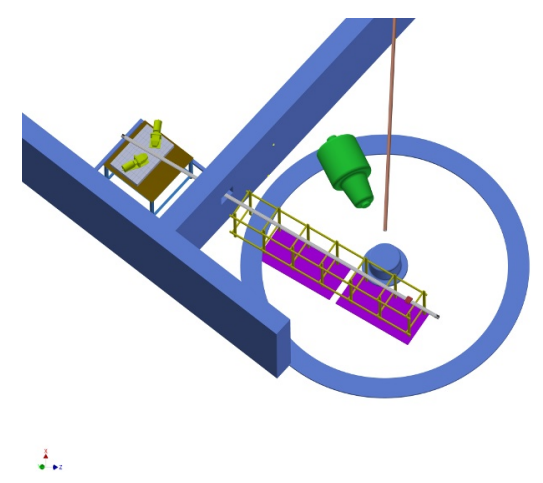

Figure 2. A schematic of the $10 \mathrm{~m}$ RABITTS. The track extends from the counting station with two HPGe detectors on the left side, through the concrete shielding wall, to the irradiation position on the right side. 
A new digital data acquisition (DAQ) system was utilized for this project. All signals are recorded in list-mode electronics and time stamped with sub-microsecond precision. This includes the HPGe detectors, fission chambers, servo motor movements, a liquid scintillator neutron flux monitor, and a beam current indicator. The system allows for $\gamma-\gamma$ coincidence analysis between the HPGe detectors. The servo motor controller is programmed to output a signal when the target leaves and arrives at each position. Thus every event can be precisely tagged in the activation cycle.

The RABITTS is also used to pulse the incident neutron beam. The servo motor is programmed to control the voltage supply of a steerer magnet located in the transport section of the charged particle beam ( $p$ or $d$ ). This steerer deflects the beam so no neutrons are produced during the counting and transit periods. This procedure provides a sharp cut off for the irradiations, ensures no additional irradiation occurs in transit, and reduces any neutron-induced backgrounds observed in the HPGe detectors. The neutron flux is measured using a liquid scintillator neutron detector downstream of the target. During counting and transit cycles, this detector measures background rates. After the target returns to the irradiation position, the neutron flux will return to is maximum value over $50 \mathrm{~ms}$. Although this time is very fast compared to our measured $t_{1 / 2}$, the beam rise time is accounted for as a beam fluctuation correction in our analysis procedure. This system is fully synchronized by design, and is not sensitive to drifts between the DAQ, servomotor, or beam pulsing system. The DAQ system was used to measure the total cycle time over 1000 cycles, and determined a total deviation of $<0.2 \mathrm{~ms}$. Nonetheless, we measure and correct for this small timing deviation during analysis.

\section{Testing the system}

Prior to measurements of fission spectra, an extensive campaign was undertaken to benchmark and characterize the RABITTS. This ensures our FPY values will be of highest quality and fidelity. The system was calibrated using standard $\gamma$-ray calibration sources, such at ${ }^{137} \mathrm{Cs}$. To test the performance of the system with beam, we measured neutron-induced isomer production. The $t_{1 / 2}=7.75 \mathrm{~s}{ }^{197 m} \mathrm{Au}$ isomer was first measured. The strong characteristic $\gamma$-ray transitions from this source are clearly seen (Fig. 3), following irradiation with $E_{n}=2.0 \mathrm{MeV}$ neutrons. The preliminary measured $t_{1 / 2}=7.73 \pm 0.06 \mathrm{~s}$ agrees with the literature value [10].
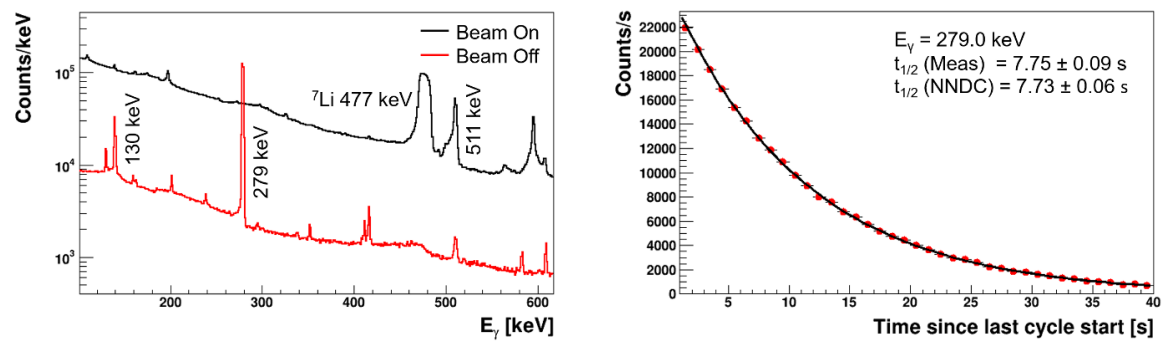

Figure 3. Measurement of the ${ }^{197} \mathrm{Au}\left(n, n^{\prime}\right){ }^{197 m} \mathrm{Au}$ reaction. The measured $\gamma$-ray spectra is shown with the characteristic peaks at 130.2 and $297.01 \mathrm{keV}$. Note the reduction in background between the irradiation and counting cycles due to the beam pulsing system. The decay curve of ${ }^{197 m} \mathrm{Au}$ is also shown, with a preliminary $t_{1 / 2}=7.73 \pm 0.06 \mathrm{~s}$ 
These data were taken with the $1 \mathrm{~m}$ RABITTS. Although the HPGe detectors are well shielded, they are sensitive to the large neutron flux, as seen in the beam on and beam off spectra of Fig. 3. The effectiveness of the beam pulsing system may be seen between the two spectra. By moving to the $10 \mathrm{~m}$ RABITTS, the issue is further alleviated, reducing any residual activation in the HPGe detector components.

To test the lower limits of the RABITTS, the $t_{1 / 2}=809 \mathrm{~ms}{ }^{90 m} \mathrm{Zr}$ isomer was also studied. Here the isomer life time closely matches the transit time, providing a good sensitivity measurement. Performing cyclic activation with the $1 \mathrm{~m}$ RABITTS at $E_{n}=5.0 \mathrm{MeV}$ produced the $\gamma$-ray spectra and decay curve shown in Fig. 4 . The preliminary measured $t_{1 / 2}=787 \pm 8$ $\mathrm{ms}$ is notably shorter than the literature value of $t_{1 / 2}=809 \pm 2 \mathrm{~ms}$ [11].
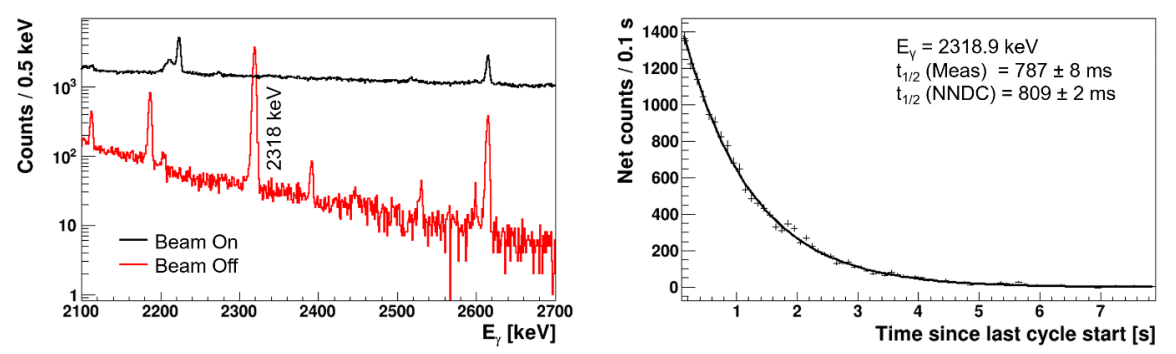

Figure 4. Measured $\gamma$-ray spectra showing the 2186.2 and $2318.9 \mathrm{keV}$ transitions from the decay of ${ }^{90 m} \mathrm{Zr}$, and the resulting decay curve of the $2318.9 \mathrm{keV} \gamma$-ray yield.

\subsection{First fission data}

Immediately following the previous isomer production tests, we proceeded with measurements of fission spectra using RABITTS. Focusing on short-lived fission products, strong $\gamma$ rays from the decay of ${ }^{96} \mathrm{Sr}\left(t_{1 / 2}=1.07 \mathrm{~s}\right),{ }^{99} \mathrm{Zr}\left(t_{1 / 2}=2.1 \mathrm{~s}\right)$, and ${ }^{97 m} \mathrm{Y}\left(t_{1 / 2}=1.17 \mathrm{~s}\right)$ were immediately observed. The ${ }^{97 m} \mathrm{Y}$ decay is shown in Fig. 5 using the $10 \mathrm{~m}$ RABITTS with a $4 \mathrm{~s}$ irradiation, $1.1 \mathrm{~s}$ transit, and $8 \mathrm{~s}$ counting interval. The short $t_{1 / 2}=1.17 \mathrm{~s}$ fission product is still strongly observed, even given the relatively long $1.1 \mathrm{~s}$ transit time associated with the $10 \mathrm{~m}$ track. Cycle times were adjusted to investigate fission products with $t_{1 / 2}$ from 1 to $300 \mathrm{~s}$. During the FPY measurements, cyclic activation of actinide targets were conducted during the day, and an $>8 \mathrm{~h}$ counting cycle was performed overnight. This overnight counting period helps to reduce activity from long-lived fission product build up in the target. Furthermore the decays from the long-lived fission products that have been produced in the irradiation are also measured by the HPGe detectors, resulting in measured yields for products with $t_{1 / 2}<8 \mathrm{~h}$. This permits us to seamlessly connect very short (independent) yields with long-lived (cumulative) yields in the same experiment.

\section{Conclusions}

Over $300 \gamma$-ray lines, representing over 50 unique fission products, have been observed by performing cyclic activation with RABITTS. Data has been taken on ${ }^{235,238} \mathrm{U},{ }^{239} \mathrm{Pu}$ for neutron-induced fission at $E_{n}=2.0,4.5 \mathrm{MeV}$, and photofission at $E_{\gamma}=11.2,13.0 \mathrm{MeV}$. Analysis on these data sets is currently underway. Future plans include the incorporation of the fission induced electromagnetic response (FIER) [12] code to investigate fission products 


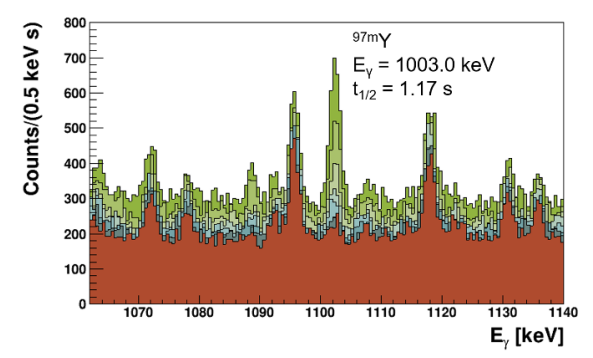

Figure 5. A measured $\gamma$-ray spectra taken using cyclic activation of ${ }^{238} \mathrm{U}(n, f)$ at $E_{n}=2.0 \mathrm{MeV}$, with $4 \mathrm{~s}$ irradiation, $1.1 \mathrm{~s}$ transit, and $8 \mathrm{~s}$ counting time. Each histogram represents a $1 \mathrm{~s}$ counting interval. The strong $1003.0 \mathrm{keV}$ line results from the decay of ${ }^{97 m} \mathrm{Y}+{ }^{97} \mathrm{Y}$, with half-lives of 1.17 and $3.75 \mathrm{~s}$, respectively.

and extract yields. We have demonstrated the ability to measure yields of fission products with $t_{1 / 2}$ from $1 \mathrm{~s}$ to $300 \mathrm{~s}$. These data will connect to our previously measured cumulative FPYs of $t_{1 / 2}>300 \mathrm{~s}$ to produce a complete, self-consistent nuclear data set. RABITTS is a new capability added to the experimental facilities at TUNL. In addition to its primary objective of measuring FPYs, RABITTS is a robust system for measuring neutron or $\gamma$-induced nuclear reactions that product radioactive products. It allows us to compare FPY data from two different nuclear reactions, where both mechanisms are studied under the same experimental conditions. It will extend our fundamental knowledge of nuclear fission by providing highly accurate FPY data for theoretical models using two electrically neutral incident particles: the neutron and photon, which induce fission via the strong nuclear interaction and by electromagnetic stimulation, respectively.

\section{Acknowledgments}

This work was supported in part by the National Nuclear Security Administration Stewardship Science Academic Alliances grant no. DE-NA0003884, DE-NA0003887, and the U.S. Department of Energy, Office of Nuclear Physics, under grant no. DE-FG0297ER41033. The work was performed under the auspices of the US Department of Energy by Los Alamos National Laboratory and Lawrence Livermore National Laboratory under Contract No. DE-AC52-07NA27344. We would like to thank Todd Bredeweg and Krishichayan for their assistance to this project.

\section{References}

[1] C. Bhatia et al., Phys. Rev. C 91, 064604, (2015)

[2] M. Gooden et al., Nucl. Data Sheets 131, 319 (2016)

[3] T.R. England and B.F. Rider, "Evaluation and compilation of fission product yields," ENDF-349, LA-UR-94-3106 (1994)

[4] Krishichayan et al., Phys. Rev. C 100, 014608, (2019)

[5] H.R. Weller et al., Prog. Part. Nucl. Phys. 62, 275 (2009)

[6] C. Bhatia et al., Nucl. Instr. Meth. Phys. Res. A 757, 7 (2014)

[7] W.W. Givens, W.R. Mills, and R.L. Caldwell. Nucl. Instr. Meth. 80, 95 (1970)

[8] N.M. Spyrou. J. Radioanal. Chem. 61, 211 (1981) 
[9] B. Pierson et al., Nucl. Data Sheets 139, 171 (2017)

[10] H. Xiaolong and Z. Chunmei, Nucl. Data Sheets 104, 283 (2005)

[11] E. Browne, Nucl. Data Sheets 82, 379 (1997)

[12] E.F. Matthews et al, Nuclear Inst. and Methods in Physics Research, A 891, 111 (2018) 